\title{
Prolongation of Ex Vivo-Perfused Pig Xenograft Survival by the Complement Inhibitor Compstatin
}

\author{
A.E. Fiane, T.E. Mollnes, V. Videm, T. Hovig, K. Høgåsen, O.J. Mellbye, L. Spruce, W.T. Moore, A. Sahu, \\ and J.D. Lambris
}

$\mathrm{T}$

HE COMPLEMENT system has been shown to play a central pathophysiologic role in hyperacute rejection $(\mathrm{HAR})^{1}$ and to contribute to the inflammation and organ injury associated with transplantation. ${ }^{2}$ Recently, a novel phage-displayed C3-binding peptide (Compstatin) has been identified that suppresses complement activation and therefore may be of therapeutic value in clinical situations such as xenotransplantation that involve complement-mediated tissue damage. This peptide binds reversibly to the $\mathrm{C} 3 \mathrm{c}$ portion of native C3 and inhibits both the classical and alternative pathways of complement activation. ${ }^{3}$ Our results suggest that inhibition of complement can be achieved with Compstatin and that this inhibition can significantly prolong xenograft survival.

\section{MATERIALS AND METHODS}

Six Norwegian Landrace pigs were used. Kidneys were removed using sterile procedures, and the ex vivo perfusion system has previously been described in detail. ${ }^{4}$ Compstatin (ICVVQDWGHHRCT$\mathrm{NH}_{2}$ ), a cyclic peptide of $1551 \mathrm{D}$, has been extensively described previously. ${ }^{3}$ Heparinized fresh $\mathrm{AB}$ whole blood was drawn from informed, healthy human volunteers. Conditions of oxygen, carbon dioxide, electrolyte content, $\mathrm{pH}$, and temperature were allowed to stabilize before perfusion began, thus imitating a clinical situation.

Compstatin-perfused circuit $(n=6)$ : Pig xenografts were perfused with human blood containing Compstatin (final conc 0.088 $\mathrm{mg} / \mathrm{mL})$. In the control group $(\mathrm{n}=6)$, pig kidneys were perfused with human blood and control agent. The total fluid volume, protein concentration, and amount of blood were equal in both the Compstatin and control perfusion circuits, and perfusion was performed simultaneously in both circuits with pooled blood from the same two donors. The flow rate at the start of the perfusion was maintained at 50 to $100 \mathrm{~mL} / \mathrm{min}$, and the mean arterial pressure (MAP) was 80 to $120 \mathrm{~mm} \mathrm{Hg}$ in all experiments. Rejection was defined as a $100 \%$ increase in vascular resistance and was characterized by marked macroscopic pathologic changes, with patchy haemorrhage and swelling of the perfused kidneys. Graft survival time was defined as the time elapsed from start of perfusion until rejection. Samples were collected from the perfusion circuits before the addition of Compstatin or control agent, after the addition of these agents at 5, 15, 60, and 180 minutes after the start of perfusion, and at the time of rejection.

\section{RESULTS}

The survival of the Compstatin-perfused kidneys (median $=380$ minutes $)$ was significantly $(P=.0036)$ longer than that of the controls (90 minutes). The classical complement pathway (C1rs-C1 inhibitor complexes and $\mathrm{C} 4 \mathrm{bc}$ ) was significantly and equally activated in both groups during the first 60 minutes. C3 activation products increased fivefold and terminal complement complex (TCC) eightfold in the control group, but no increase occurred in the Compstatin group during this period. Immunohistochemistry showed less C3 and fibrin deposition and immunoelectron microscopy showed less TCC deposition in the Compstatin group. A significant change in total white cells, neutrophils, myeloperoxidase, and expression of the surface activation markers CD11b (CR3) and CD35 (CR1) and CD62L (L-selectin) was observed in both groups. A trend toward less leukocyte activation as measured by myeloperoxidase was found in the Compstatin group $(P=.08)$. There were no differences in platelet counts, thrombospondin, soluble CD62P (soluble P-selectin), or $\beta$-thromboglobulin (BTG) between the groups.

\section{DISCUSSION}

The present study indicates that the use of Compstatin to inhibit complement activation may significantly prolong

From the Department of Surgery A (A.E.F.), Department of Pathology (T.H.), and Institute of Immunology and Rheumatology (K.H., O.J.M.), University of Oslo, The National Hospital, Oslo, Norway; Department of Immunology and Blood Bank, Norwegian University for Science and Technology, Trondheim, Norway (V.V.); Department of Immunology and Transfusion Medicine, Nordland Central Hospital, and University of Tromsø, Bodø, Norway (T.E.M.); and Laboratory of Protein Chemistry, Department of Pathology and Laboratory Medicine, University of Pennsylvania, Philadelphia, Pennsylvania, USA (L.S., W.T.M., A.S., J.D.L.).

Supported by the Nanki Bergesen and Wife's fund, the Norwegian Council on Cardiovascular Disease, National Institute of Health Grants Al 30040 (to J.D.L.), and by Cancer and Diabetes Centers' Core Support Grants CA16520 and DK 19525.

Address reprint requests to Dr Arnt E. Fiane, Surgical Department A, Rikshospitalet, Pilestredet 32, N-0027 Oslo, Norway. 
graft survival and reduce HAR of porcine kidneys perfused with human blood. As expected, classical complement activation, indicated by the levels of $\mathrm{C} 1$ and $\mathrm{C} 4$ activation products, was similar in the two groups, because Compstatin inhibits at a later step in the cascade. The significantly greater increase in the concentrations of $\mathrm{C} 3 \mathrm{bc}$ and TCC in the control group than in the Compstatin group indicates that Compstatin did indeed act at the level of $\mathrm{C} 3$. The fluid-phase complement data were supported by immunohistochemistry and electron microscopy studies showing less C3 and TCC deposition, respectively. Furthermore, the urinary data strongly suggest that Compstatin efficiently protects the kidney from complement-mediated damage. The number of leukocytes, neutrophils, and monocytes decreased significantly in both groups, indicating increased cell adhesion, extravasation, and/or destruction during perfusion.

Flow cytometry analysis revealed inflammatory response-related changes in a number of surface adhesion molecules on neutrophils in both Compstatin and control setups: $\mathrm{CD} 11 \mathrm{~b}$, the receptor for $\mathrm{iC} 3 \mathrm{~b}$, was markedly upregulated; $\mathrm{CD} 35$, a receptor for $\mathrm{C} 3 \mathrm{~b}$ that also acts as a cofactor for factor I-mediated C3b cleavage, was moderately upregulated; and CD62L was downregulated. Studies in humans have indicated that upregulation of CD11b and downregulation of CD62L on neutrophils are related to endothelial changes leading to capillary leakage. We observed a trend toward lower myeloperoxidase release in the Compstatin group than in the control group at up to 60 minutes. However, the other leukocyte activation markers studied (number of leukocytes, CD11b, CD35, CD62L) were not statistically different between the two groups.

Whether Compstatin had a modulatory effect on these inflammatory cells by indirectly attenuating complement activation is therefore unclear.

The number of platelets decreased significantly by time from start to rejection in both groups, and microthrombi were observed in small vessels in both groups at rejection by electron microscopy, consistent with the role of thrombosis as an important process in HAR. However, no difference in the number of platelets or in the fluid-phase activation markers soluble CD62P, BTG, or thrombospondin was found between the groups.

We conclude that Compstatin prolongs graft survival and suggest that it may be a useful agent for attenuating HAR by inhibiting $\mathrm{C} 3$ and thus terminal complement pathway activation.

\section{REFERENCES}

1. Dalmasso AP: Immunopharmacology 24:149, 1992

2. Baldwin WM III, Pruitt SK, Brauer RB, et al: Transplantation 59:797, 1995

3. Sahu A, Kay BK, Lambris JD: J Immunol 157:884, 1996

4. Fiane AE, Videm V, Mollnes TE, et al: Transplant Proc 27:3560, 1995

5. Elliot MJ, Finn AH: Ann Thorac Surg 56:1503, 1993 